\section{Ampliando oportunidades para promover funcionalidad y prevenir la dependencia en población adulta mayor}

\section{Expanding opportunities to promote functionality and prevent dependency in older adults}

\section{Sr. Editor}

La mantención de la funcionalidad y prevención de la dependencia en adultos mayores constituye un objetivo estratégico de las Metas Sanitarias de la Década 2011-2020. Ante esto, el sector salud desarrolla variadas estrategias, como el Examen de Medicina Preventiva del Adulto Mayor (EMPAM) en Centros de Salud de Atención Primaria (APS), que incluye la Evaluación Funcional del Adulto Mayor (EFAM), reconocido como garantía explícita de salud. EFAM busca evaluar la funcionalidad para identificar el riesgo de dependencia y la fragilidad ${ }^{1}$. Las metas sanitarias delimitan la fragilidad como un constructo complejo con dimensiones biológica, funcional, y clínica que aporta con un enfoque de riesgo conveniente para la planificación en salud.

Desde el año 2015 se desarrolla además el programa Más Adultos Mayores Autovalentes, que busca mejorar y/o mantener la condición funcional y la estimulación funcional a través de organizaciones sociales locales, para personas de 60 años y más cuyo EMPAM les categorice como autovalente sin riesgo, o autovalente con riesgo o en riesgo de dependencia. El Programa se centra en estimulación de funciones motores, cognitivas, y el autocuidado y educación en salud, junto con la capacitación a la comunidad. La efectividad del programa es evaluada según su resultado en la funcionalidad física, con el instrumento Health Assesment Questionnaire-8 (HAQ-8) (González et al, 1995), que evalúa mediante autoaplicación la percepción de dificultad en realización de actividades diarias. Ha sido validado en población con atritis reumatoide y fue utilizado en la Encuesta Nacional de Salud 2003, sin una validación específica en población adulta mayor según las categorías del EMPAM.

Si bien debe resaltarse el evidente compromiso institucional ante esta prioridad de salud pública, resulta oportuno considerar elementos de actual discusión científica en torno al problema de la fragilidad y la prevención de dependencia en población adulta mayor, identificando oportunidades para las actuales definiciones técnicas.

Una reciente revisión de literatura sobre criterios de fragilidad en población mayor ${ }^{2}$ propone la consideración de un perfil de fragilidad en adultos mayores que involucre las dimensiones de fragilidad física, psicológica, y social, según los siguientes criterios: a. Fragilidad física: Pérdida de peso involuntario, fuerza muscular, cansancio físico, percepción de estado de salud, equilibrio, y problemas sensoriales (visión y audición).

b. Fragilidad psicológica: Cognición, síntomas depresivos, ansiedad, y estrategias de enfrentamiento.

c. Fragilidad social: Vivir solo, relaciones sociales y soporte social.

Según la revisión, la fragilidad física y psicológica tendrían un mayor respaldo, no así la fragilidad social, por lo que se insta a investigar e intervenir en esta dimensión, resaltando el reconocimiento de la fragilidad en sus dimensiones psicológicas y sociales, y la relación transaccional entre los tres ámbitos de fragilidad.

La salud pública ha elevado una creciente crítica al protagonismo que ha tenido la función física y cognitiva como elementos centrales en la prevención y tratamiento de la pérdida funcional, en desmedro de otras dimensiones de la fragilidad. Desde un marco de determinación social de la salud, se enuncia la complejidad de la funcionalidad como resultado de salud, su prevención y promoción, que da cuenta de la inequidad en que las trayectorias de funcionalidad se dan en Chile. Se ha señalado, por ejemplo, la estratificación social de la pérdida funcional en adultos mayores chilenos a partir del efecto protector de los recursos económicos y educacionales, sugiriendo además que la edad no actúa como nivelador de dicho efecto ${ }^{3}$.

En coherencia, teniendo como referencia el marco para el Envejecimiento Saludable y los Determinantes Sociales de la Salud, Sadana et al $(2016)^{4}$ esquematizan la determinación de la salud de la población adulta mayor (Figura 1), proponiendo la funcionalidad como un resultado de salud con variados determinantes y moduladores.

Dicho análisis considera el Envejecimiento Saludable debido a que el marco de envejecimiento activo ha tenido una limitada transferencia a las prácticas en salud al tratarse de un marco político global, que ha favorecido el imaginario de la actividad física como resultado central de la promoción de salud en adultos mayores ${ }^{5}$.

Según lo descrito, resulta oportuno examinar los constructos de referencia y las estrategias que se desarrollan actualmente para la prevención de dependencia y abordaje de la fragilidad en adultos mayores. La focalización de las actuales políticas en dimensiones de fragilidad y funcionalidad más bien físicas, con elementos incipientes desde dimensiones psicológicas, y en menor manera, sociales.

Es necesario también explorar el potencial de disciplinas no tradicionalmente vinculadas con la rehabilitación. Por ejemplo, existe evidencia sobre la asociación de la salud oral con deterioro cognitivo ${ }^{6}$, un mediador de dependencia no tradicionalmente considerado en fragilidad y funcionalidad físicas, ¿sería pertinente incluir al profesional odontólogo(a) en la 


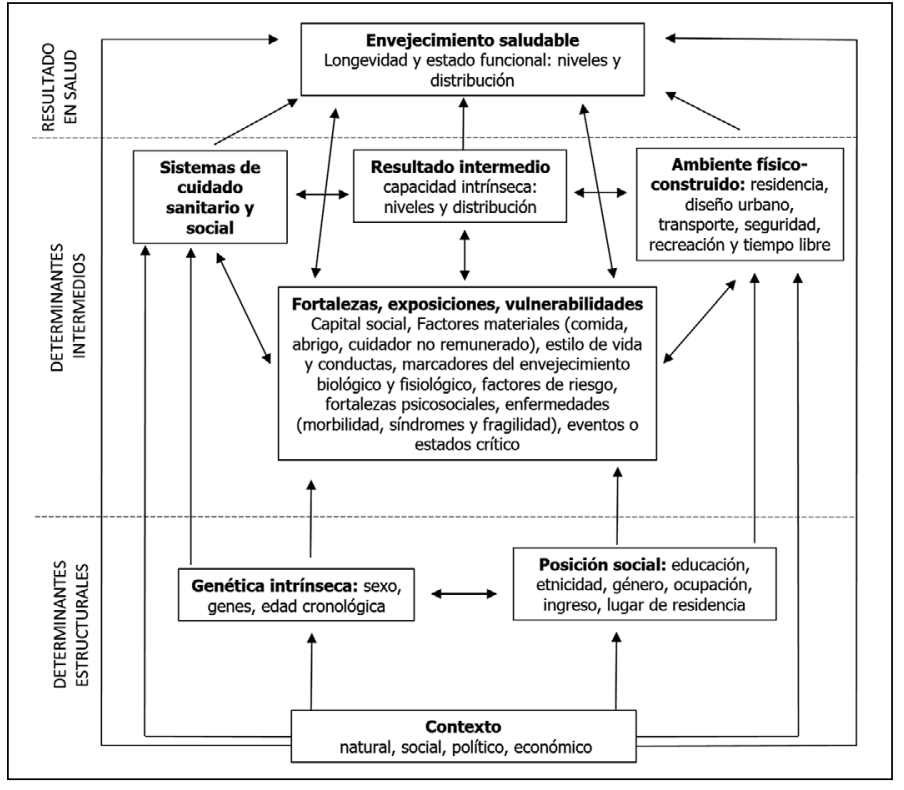

Figura 1. Factores que contribuyen a los niveles y distribución de la salud en la adultez mayor. Fuente: Adaptado de Sadana et al (2016). promoción de funcionalidad cognitiva y prevención de dependencia? Luego, emerge la necesidad de delimitar los modelos teóricos que sustenten el contenido de las intervenciones. Esto favorecería otro aspecto crítico de examinar en las actuales intervenciones preventivas de dependencia: su escasa estandarización y evaluación de validez y efectividad en Chile.

Finalmente, debido a que el principal resultado de la meta sanitaria se traduce en la disminución de un $10 \%$ en la media de puntaje de discapacidad física en población de 65 a 80 años, podría ser considerada una mayor variedad de resultados no sólo de funcionalidad física. La actualización de orientaciones técnicas es una oportunidad al corto y mediano plazo, favoreciendo el tránsito de los programas preventivos hacia intervenciones multicomponente que consideren las dimensiones sociales y psicológicas de la fragilidad. En un largo plazo, la funcionalidad y dependencia debiesen constituir resultados de salud abordados por medio de acción intersectorial.

Jean Gajardo ${ }^{1, a}$

${ }^{1}$ Departamento de Terapia Ocupacional y Ciencia de la Ocupación, Facultad de Medicina, Universidad de Chile.

${ }^{a}$ Candidato a Doctor en Salud Pública. Master en Gerontología Social.

\section{Referencias}

1. Ministerio de Salud de Chile. Orientación técnica para la atención en salud de las personas adultas mayores en Atención Primaria. 2014.
2. Iriarte E, Araya A-X. Criterios de fragilidad en personas mayores que viven en la comunidad: una actualización de la literatura. Rev Med Chile [Internet]. 2016; 144: 1440-7. Disponible en: http://revistamedicadechile.cl/ ojs/index.php/rmedica/article/view/4758

3. Fuentes-García A, Sánchez H, Lera L, Cea X, Albala C. Desigualdades socioeconómicas en el proceso de discapacidad en una cohorte de adultos mayores de Santiago de Chile. Gac Sanit. 2013; 27 (3): 226-32.

4. Sadana R, Blas E, Budhwani S, Koller T, Paraje G. Healthy Ageing: Raising awareness of inequalities, determinants, and what could be done to improve health equity. Gerontologist. 2016; 56: S178-93.

5. Organización Mundial de la Salud. Informe mundial sobre el envejecimiento y la salud [Internet]. 2015. Disponible en: http://www.who.int/ageing/publications/ world-report-2015/es/

6. Wu B, Fillenbaum GG, Plassman BL, Guo L. Association between Oral Health and Cognitive Status: A Systematic Review. J Am Geriatr Soc 2016; 64 (4): 739-51.

Esta carta se enmarca en el estudio FONIS SA1610137, financiado por la Comisión Nacional de Investigación Científica y Tecnológica del Gobierno de Chile-CONICYT.

Correspondencia a:

Jean Gajardo

Profesor Asistente, Departamento de Terapia Ocupacional y Ciencia de la Ocupación, Facultad de Medicina, Universidad de Chile. Av. Independencia 1027. Comuna de Independencia, Santiago. jean.gajardo@uchile.cl 